
\title{
25 Research Soure \\ Family physician program and burden of referral for outpatient visit in Iran: A multicenter cross-sectional study protocol
}

Mobin Sokhanvar

Tabriz University of Medical Sciences

Mohammad Javad Kabir

Golestan University of Medical Sciences and Health Services School of Health and Paramedicine Hossein Bevrani

Tabriz University of Medical Sciences

Shirin Nosratnejad

Tabriz University of Medical Sciences

Ali Janati

Tabriz University of Medical Sciences

Morteza Arab-zozani

Birjand University of Medical Sciences

Edris Hasanpoor ( $\nabla$ edihasanpoor@gmail.com )

Maragheh University of Medical Sciences https://orcid.org/0000-0002-5155-2679

\section{Study protocol}

Keywords: Family physicians Program, burden, referral, outpatients visit, Iran

Posted Date: January 10th, 2020

DOI: https://doi.org/10.21203/rs.2.20540/v1

License: (c) (i) This work is licensed under a Creative Commons Attribution 4.0 International License. Read Full License 


\section{Abstract}

Background: Primary healthcare (PHC) developed around the world with a focus on the family physician, which can improve access and quality of services. The study will estimate the burden of referral for outpatients visit through a family physician program (FPP) in Iran.

Methods : This will be a descriptive-analytical cross-sectional study. The data for self-referrals and those who are officially referred to the specialized level of outpatient care will collect from the rural and urban populations in four provinces. Of these provinces, two are the pilot sites of urban FPP and the other two provinces are matched provinces. Patient recruitment will be carried out by a research assistant located in each health network of the province. We will collect the data on outpatient visits, prescribed medicine, medical imaging, and laboratory services. Descriptive and analytical statistics will be generated to estimate the burden of referral to outpatient visits among and between provinces.

Discussion : This study has been approved by the Ethics committee of Tabriz University of Medical Sciences. The results of this study will be the first national evidence of the effectiveness of FPP and the burden of referral for outpatients visit in Iran. One of the main strategies to improve access, quality and efficiency of healthcare systems is a family physician program (FPP) as the primary care provider within an appropriate referral system. Evidence suggests that health systems, with a focus on PHC, have had positive consequences for health outcomes, healthcare quality, access to and continuity of health care, system efficiency and financial sustainability and public satisfaction.

\section{Background}

Health is a universal right and providing health services is a government's responsibility [1]. Health systems are created to provide affordable and accessible health care for all people and to make this dream come true. One of the designs that brought us closer to this goal was the primary healthcare system (PHC). [2, 3]. The PHC was introduced as an acceptable, accessible and possible strategy for national health systems regarding health promotion and as a route to realizing sustainable social and economic development [4].

\section{Primary healthcare \& Family Physician Program (FPP) in Iran}

Concurrent with the Iranian Revolution in 1979, some major changes were made to promote social justice. In this regard, significant changes were made as a provider of health services using establishing and expanding health networks in 1984 [5]. In the Iranian health network system, health services are provided at three levels. The first level relates to PHC. The so-called health houses are considered to be the starting point for serving rural communities. Those which are run by community health workers are known as Behvarz. These centers have proven to be very effective in improving health indicators $[5,6]$. In larger settlements, in addition to health houses, there are Rural Health Centers (RHCs). The RHCs staff 
includes a general physician and a team of up to 10 health workers. In urban areas, health services are provided by health posts and Urban Health Centers (UHCs). All rural and urban centers are managed by district health centers, under the supervision of the Medical Sciences University. In urban areas, with a population of more than 50000 , there is at least one general hospital and a polyclinic that provides secondary care level. There is one Medical Sciences University per province. There are teaching hospitals in each province capital providing specialized and sub-specialized services as the third level of healthcare services under the supervision of Medical Universities [7].

Family physician plan (FPP) was launched in the Iranian health system in rural and urban areas with a population below 20,000in 2005. [8]. Since it was emphasized in the fourth national strategic program on Iran's economy, social and cultural development to expand the coverage of health insurance with a significant focus on family physician and referral system, it was planned to extend the implementation of the plan to large urban areas. According to the law of the fifth development plan, approved in 2010, the Iranian Health Insurance Organization (IHIO) was obliged to comply with the referral system and was designated as the main implementer of the FPP [9]. The IHIO is one of Iran's main insurance organizations covering $41 \%$ of the total population, including residents of rural areas [10].

Accordingly, since the 8th of July 2012, the FPP has been running as a pilot project in urban areas of two provinces, Fars and Mazandaran [11]. According to the Iranian FPP and in line with the referral system instructions for rural and urban areas, participating family physicians are responsible for people's primary care and follow-up of patients who have been referred to the specialized and sub-specialized levels [12]. The referral system consists of all physicians included in FPP, regardless of whether they work in the private or public sector, at the second and third levels. The second and third levels of the provision of services in Iran are obligated to providing specialized services for patients who had been referred by the first level [13]. Adherence to the referral system is not mandatory in Iran. Some people are directly referred to the specialized levels and bypass the referral system, the process known as self-referral. In this case, the fees are slightly higher than those whose referrals were processed through the referral system [14].

\section{Family Physician extent and consequences}

To deliver basic health care in the form of the gatekeeping concepts [15], primary care physicians in various countries are employed by health systems. Gatekeeping is common in Europe, both in tax-funded and in social health insurance systems. Gatekeeping in the United States is prevalent, especially in the context of managed care $[16,17]$. Quality, accessible and cost-effective health services as well as the satisfaction of end-users, are the core principles in any health care system, following through family physicians and referral systems [18]. Six pathways have been introduced by Starfield et al. (2005), to illustrate the positive effects of having a primary care physician on health outcomes including greater access to needed services, better quality of care, greater focus on prevention, early management of health problems, cumulative effect of the main primary care delivery characteristics, and the role of primary care in reducing unnecessary specialist care [19]. 
The FPP emerged and was developed with its primary goal of managing and rationalizing health services utilization [20]. Health systems whose main focus is to provide primary care through family physicians, reduce unnecessary specialized services through enhancing the continuity and coordination of care [19]. The results of a systematic review revealed the positive consequences of family physician gatekeeping in terms of reduction in health services utilization and economic burden by 78 and 80 percent, respectively [16]. Ma et al. (2016) examined the utilization of services and their costs in patients diagnosed with arthritis, which had enrolled in gatekeeper and non-gatekeeper plans. The results revealed that gatekeeper health plans are associated with lower health services (office-based medical visits, hospital inpatient visits, outpatient visits, and prescription medications) use and expenditures [21]. The results of other studies have shown that having a primary care physician was associated with lower utilization of specialists, emergency rooms [22] as well as receipt of a variety of preventive health care checks including blood pressure, cholesterol, blood sugar, blood stool test, and reduced number of those receiving flu shot services [23].

In Iran, despite the long history of implementing the FPP, there are very few studies on the effectiveness of FPP in services utilization management. Based on the view of Heshmati and Joulaei (2016), FPP in Iran has not been well implemented and the option of self-referring to specialists still exists [24]. A survey among 500 households living in urban and rural areas of Fars province showed, that direct referral to a specialist physician while disregarding the health problem, was common among approximately 60 percent of participants [14]. Furthermore, Barati et al. (2016) showed that, after the implementation of FPP in urban areas in Shiraz (The biggest city of Fars province), utilization of Para-clinic services has decreased by 4 and 19 percent in public and private centers, respectively [25]. Also, high inequalities of specialist visits' utilization were revealed in Iran [26].

The Iranian FPP and referral system have longstanding challenges that may affect its function in terms of service utilization management. Lack of interaction and constructive communication between the three levels of service delivery, disagreement among service providers regarding the effectiveness of this program, diversity of basic insurance plans, absence of complete public awareness, lack of compulsion on the way of receiving service and a small difference in payment on different routes, as well as limited IT infrastructure to create electronic health record are just some of the challenges they face $[13,27,28]$.

A lack of comprehensive evidence in this area is another challenge policy-makers are facing. Pilot field studies have generally been conducted without comparison to other environments as a measure of control $[14,25]$. Accordingly, this study will design to extract evidence on the effects of FPP on the burden of referral for outpatients visit through family physician program (FPP) in Iran.

\section{Methods}

\section{Study design}


In this multicenter descriptive-analytical cross-sectional study, the burden of referral for an Outpatient visits in rural areas and cities with a population of less than 20,000 as well as urban areas with a population of more than 20,000 will be estimated. FPP performance rates in rural areas will be determined by comparing utilization rates of self-referred patients with people who had been referred through the referral system and based on the family doctor's order. In urban areas, the effectiveness of FPP is determined by comparing the rates of utilization of outpatient services in provinces of Fars and Mazandaran (pilot sites of urban FPP implementation) with matched provinces.

\section{Setting}

The FPP program in rural and urban areas with population under 20,000 has been nationally implemented since 2005. Due to capabilities of the research team, such as access to data and financial resources, the same provinces were selected for comparing utilization rate in rural and urban populations. In this regard, the provinces of Fars and Mazandaran were purposefully selected due to the pilot implementation of FPP in urban areas. Clustering techniques, based on a set of indicators, including urban and rural population covered by IHIO, were used to select the matched provinces, the number of general practitioners, the number of specialist and subspecialist doctors, the number of medical centers on the contract with $\mathrm{IHIO}$, the economic participation rate of the province, the employment rate, the rate of being literate, the annual cost and income of the rural and urban households. The Rapid miner software will use along the K-means clustering method for the purpose of this research (Rapid Miner version 4.1 Beta 2, 2001-2007). In this study, the Davis-Bouldin Index will use to validate clustering. This indicator determines the number of clusters showing the best distribution [29]. Based on the results, the most favorable one was the number of four clusters which highest D-BI score. By selecting the number of clusters by 4, Fars and Mazandaran provinces, along with 6 other provinces (East Azerbaijan, West Azerbaijan, Esfahan, Khuzestan, Gilan, and Khorasan Razavi) were located in the same cluster and the remaining 23 provinces were located in the three other clusters. From the remaining six provinces, located in the same cluster with Fars and Mazandaran, the two will be randomly selected.

\section{Study population and sampling}

The entire population of the four selected provinces, insured by the IHIO, will be targeted. The population covered by the IHIO will be identified through the users' database for each selected province. It will then enroll 1066 persons from rural residents and the same number from urban residents to achieve 95 percent confidence with a 0.03 error in statistical analyses based on the insured population of each province. At each study sites, the number of samples will be divided equally between the two groups of self-referrals and patients referred by family physicians. Figure 1: summarizes the sample selection approach in the provinces. Selection of the samples from the study population will be done by means of a systematic randomization approach through the insurance code of individuals.

\section{Participants' eligibility and identification}

The utilization rate in this study will be extracted retrospectively for one year period, so at the time of recruitment, the age of every participant (in both groups) should be one year plus the extent passed from 
the year over which the study will be done. This inclusion criterion assured a minimum age of 1 day at the beginning of the data collection period. Additionally, the use of at least one type of the selected outpatient service within the data collection period will be considered as another inclusion criteria.

There are two types of identifying factors of self-referrals and individuals with legitimate referrals. Primary factor identifies unregistered individuals in both rural and urban FPP who have received specialized outpatient care will be considered as self-referred. The secondary factor will be used to identify the two group members is financial records which registered in the IHIO information system. There is a difference between the self-referrals and the outpatients who were referred by the family physician in terms of the co-payments. For any particular service, the co-payment paid by self-referrals is more than those which paid by regularly referred outpatients.

\section{Outcomes}

In this study, all services received by outpatients will be investigated. In this regard, the main frequent outpatient services are selected, which includes specialist/sub-specialist visits, prescribed medications, and laboratory and medical imaging services. Also, the demographic variables (age, gender, place of residence and etc.) which are available in the IHIO database will be extracted.

\section{Patient and public involvement}

All participants provide written consent. Participants will be provided a summary of the finding in case of availability. Articles releases of relevant findings will inform the general population.

\section{Data analysis}

The K-means method, utilizing the Rapid miner software, was used for clustering. The SPSS-v23 software will be utilized to analyze the data and compare the rate of service utilization among the two groups. According to the type of dependent variable (discrete and non-negative), to investigate the effect of the main independent variables including the type of referral (legitimate referrals and self-referral), the study provinces and demographic variables on the utilization of services, the Poisson regression model will be used [30]. Additionally, for qualitative variables, taking into account their impact on utilization, the link diagram will be extracted by using log-liner models [31].

\section{Strength and limitations of this study}

- To our knowledge, this study will provide the first estimation of referral burden through FFP in Iran.

- The result of this study could inform policymakers, managers and general practitioners about the extent of self-referral in the Iranian healthcare system.

- Managing the data collection process and huge number of participants is one of the possible limitations.

\section{Discussion}


Undoubtedly, the results of this study will provide the first comprehensive and national evidence of the level of utilization of specialized, outpatient services, based on the type of patients' referral. This study intends to raise health policy-makers awareness of the effectiveness of FPP on outpatient utilization management. This evidence will be useful for the Iranian health system authorities in the field of reviewing and modifying the FPP and will be an enlightening experience for other countries. The predictors of the type of receiving specialist services will also be a guide for reviewing FPP, insurance policies, payment systems, and patient copayment rates.

This study is not without limitations. It will reflect the outpatient services utilization in four provinces in Iran. To make a comparison between provinces, these provinces were purpusefully selected through the process of clustering. These provinces were, therefore, almost similar in terms of healthcare infrastructure and other socio-economic indicators used in clustering. Accordingly, to generalize the results of this study to other provinces, this limitation should be considered.

\section{Abbreviations}

PHC:primary healthcare; FPP:Family Physician Program; RHCs:Rural Health Centers; UHCs:Urban Health Centers; IHIO:Iranian Health Insurance Organization.

\section{Declarations}

\section{Ethics approval and consent to participate}

Approval was not required for this study

\section{Consent for publication}

Not required.

\section{Availability of data and material}

No additional data available.

\section{Competing interests}

All authors declare no competing interests.

\section{Funding}

This research is funded by Tabriz University of Medical Sciences [grant number: 59025].

\section{Authors' contributions}


The study is designed by AJ, MS, HB and ShN. MS and AJ wrote the first draft of this manuscript. Data analysis will done by ShN and HB. MjK will facilitate data gathering from IHIO. MA-Z review and edited the final draft. All authors reviewed and approved the final manuscript.

\section{Acknowledgements}

We would like to thank all the faculty members of School of Management and Medical Informatics who reviewed the study protocol as a proposal of a doctoral dissertation.

\section{Author details}

${ }^{1}$ Iranian Center of Excellence in Health Management, School of Management and Medical Informatics, Tabriz University of Medical Sciences, Tabriz, Iran. ${ }^{2}$ Health Management and Social Development Research Center, Golestan University of Medical Sciences, Gorgan, Iran. ${ }^{3}$, Department of Statistics, Faculty of Mathematical Sciences, University of Tabriz, Tabriz, Iran. ${ }^{4}$ Iranian Center of Excellence in Health Management, School of Management and Medical Informatics, Tabriz University of Medical Sciences, Tabriz, Iran. ${ }^{5}$ Tabriz Health Services Management Research Center, Department of Health Services Management, School of Management and Medical Informatics, Tabriz University of Medical Sciences, Tabriz, Iran. ${ }^{6}$ Social Determinants of Health Research Center, Faculty of Health, Birjand University of Medical Sciences, Birjand, Iran aculty of Public Health, Institute of Health, Jimma University, Jimma, Ethiopia. ${ }^{7}$ Assistant professor of Healthcare Management, Healthcare Management, Research Center for Evidence-Based Health Management, Maragheh University of Medical Sciences, Maragheh, Iran.

\section{References}

1. Hall JJ, Taylor R. Health for all beyond 2000: the demise of the Alma-Ata Declaration and primary health care in developing countries. The Medical Journal of Australia. 2003;178(1):17-20.

2. Gillam S. Is the declaration of Alma Ata still relevant to primary health care? Bmj. 2008;336(7643):536-8.

3. Organization WH, Unicef. Primary health care: a joint report. 1978.

4. van Raak R, HAAN FJD. Key features of modern health systems: nature and historical evolution. Toward Sustainable Transitions in Healthcare Systems: Routledge; 2017. p. 62-100.

5. Shadpour K. Primary health care networks in the Islamic Republic of Iran. East Mediterranean Health Journal. 2000;6(4):822-5.

6. Farzadfar F, Murray CJ, Gakidou E, Bossert T, Namdaritabar H, Alikhani S, et al. Effectiveness of diabetes and hypertension management by rural primary health-care workers (Behvarz workers) in Iran: a nationally representative observational study. The Lancet. 2012;379(9810):47-54.

7. Tabrizi JS, Pourasghar F, Nikjoo RG. Status of Iran's primary health care system in terms of health systems control knobs: a review article. Iranian journal of public health. 2017;46(9):1156. 
8. Takian A, Rashidian A, Kabir MJ. Expediency and coincidence in re-engineering a health system: an interpretive approach to formation of family medicine in Iran. Health policy and planning. 2010;26(2):163-73.

9. Dehnavieh R, Sirizi MJ, Mehrolhassani MH, Kalantari A. Strategic purchasing of healthcare services in developing countries: A new approach is required. Iranian journal of public health. 2016;45(9):1241.

10. Mehrdad R. Health system in Iran. JMAJ. 2009;52(1):69-73.

11. Moradi-Lakeh M, Vosoogh-Moghaddam A. Health sector evolution plan in Iran; equity and sustainability concerns. International journal of health policy and management. 2015;4(10):637.

12. Honarvar B, Lankarani KB, Rostami S, Honarvar F, Akbarzadeh A, Odoomi N, et al. Knowledge and practice of people toward their rights in urban family physician program: A population-based study in Shiraz, Southern Iran. International journal of preventive medicine. 2015;6.

13. Golalizadeh E, Moosazadeh M, Amiresmaili M, Ahangar N. Challenges in second level of referral system in family physician plan: a qualitative research. Quarterly Journal of Medical Council Of IRI. 2012;29(4):309-21.

14. Niyas M, Karimi M, Kavosi Z. Utilization of Primary Health Care Services in Rural and Urban Areas in Shiraz. Shiraz E-Medical Journal (In Press). 2018;e66219.

15. Reibling N, Wendt C. Gatekeeping and provider choice in OECD healthcare systems. Current Sociology. 2012;60(4):589-05.

16. Garrido M, Zentner A, Busse R. The effects of gatekeeping: a systematic review of the literature. Scandinavian journal of primary health care. 2011;1(29):28-38.

17. Franks P, Clancy C, Nutting P. Gatekeeping revisited-protecting patients from overtreatment. The New England Journal of Medicine. 1992;327(424-429).

18. Arah OA, Klazinga NS, Delnoij DM, Asbroek At, Custers T. Conceptual frameworks for health systems performance: a quest for effectiveness, quality, and improvement. International journal for quality in health care. 2003;15(5):377-98.

19. Starfield B, Shi L, Macinko J. Contribution of primary care to health systems and health. The milbank quarterly. 2005;83(3):457-502.

20. Scott A. Economics of general practice. Handbook of health economics. 2000;1:1175-200.

21. Ma X, Park C, Lawson K. Arthritis-Related Medical Care Utilization and Expenditures Under Gatekeeper and Non-Gatekeeper Plans Using Medical Expenditure Panel Survey (Meps) Data. Value in Health. 2016;19(3):A28-A9.

22. Hurley RE, Freund DA, Taylor DE. Emergency room use and primary care case management: evidence from four Medicaid demonstration programs. American Journal of Public Health. 1989;79(7):843-6.

23. Continelli T, McGinnis S, Holmes T. The effect of local primary care physician supply on the utilization of preventive health services in the United States. Health \& place. 2010;16(5):942-51.

24. Heshmati B, Joulaei H. Iran's health-care system in transition. The Lancet. 2016;387(10013):29-30. 
25. Barati O, Pourmohammadi K, Zakeri M. Survey of the demand trend for Para-clinic services after family physician plan in Fars province. Hakim Health System Research 2016;19(1):58-63.

26. Homaie Rad E, Ghiasi A, Arefnezhad M, Bayati M. Inequalities of general physicians and specialists visits' utilization and its determinants in Iran: a population based study. International Journal of Human Rights in Healthcare. 2015;8(3):125-31.

27. Moghadam M, Sadeghi V, Parva S. Weaknesses and challenges of primary healthcare system in Iran: a review. The International journal of health planning and management. 2012;27(2):e121-31.

28. Damari B, Vosough Moghaddam A, Rostami Gooran N, Kabir M. Evaluation of the urban family physician and referral system program in Fars and Mazandran provinces: history, achievements, challenges and solutions. Journal of School of Public Health and Institute of Public Health Research. 2016;14(2):17-38.

29. Davies D, Bouldin D. A cluster separation measure. IEEE transactions on pattern analysis and machine intelligence. 1979:224-7.

30. Demidenko E. Poisson regression for clustered data. International statistical review. 2007;75(1):96113.

31. Bakeman R, Robinson BF. Understanding log-linear analysis with ILOG: An interactive approach: Psychology Press; 2013.

\section{Figures}




\section{Figure 1}

Figure 1 was not included in this version of the manuscript. 